\title{
La représentation de la communauté juive dans les manuels scolaires québécois
}

The Representation of the Jewish Community in Quebec Textbooks

\section{Representación de la comunidad judía en los textos escolares de Quebec}

\section{Sivane Hirsch et Marie Mc Andrew}

Volume 15, numéro 2, 2012

URI : https://id.erudit.org/iderudit/1018456ar

DOI : https://doi.org/10.7202/1018456ar

Aller au sommaire du numéro

Éditeur(s)

Faculté d'éducation, Université de Sherbrooke

ISSN

1911-8805 (numérique)

Découvrir la revue

Citer cet article

Hirsch, S. \& Mc Andrew, M. (2012). La représentation de la communauté juive dans les manuels scolaires québécois. Nouveaux cahiers de la recherche en éducation, 15(2), 34-63. https://doi.org/10.7202/1018456ar
Résumé de l'article

Cet article étudie la représentation de la communauté juive, de son histoire et de sa culture dans les manuels scolaires québécois de deux disciplines ayant comme objectif l'éducation des futurs citoyens avertis: Histoire et éducation à la citoyenneté et Éthique et culture religieuse. Puisque la majorité des élèves québécois ne rencontrent cette communauté que par le biais des manuels, il importe de cerner jusqu'à quel point l'enseignement qu'ils reçoivent leur permet de bien la connaître et de développer des attitudes positives à son égard. Notre étude montre bien que la communauté bénéficie désormais d'une plus grande visibilité dans les manuels, mais que celle-ci ne contribue pas toujours à une meilleure connaissance de la communauté et de son histoire au Québec. 


\section{La représentation de la communauté juive dans les manuels scolaires québécois}

\section{Sivane Hirsch}

Université de Montréal

\section{Marie Mc Andrew}

Université de Montréal

\section{La représentation de la communauté juive dans les manuels scolaires québécois}

\section{Résumé}

Cet article étudie la représentation de la communauté juive, de son histoire et de sa culture dans les manuels scolaires québécois de deux disciplines ayant comme objectif l'éducation des futurs citoyens avertis: Histoire et éducation à la citoyenneté et Éthique et culture religieuse. Puisque la majorité des élèves québécois ne rencontrent cette communauté que par le biais des manuels, il importe de cerner jusqu'à quel point l'enseignement qu'ils reçoivent leur permet de bien la connaître et de développer des attitudes positives à son égard. Notre étude montre bien que la communauté bénéficie désormais d'une plus grande visibilité dans les manuels, mais que celle-ci ne contribue pas toujours à une meilleure connaissance de la communauté et de son histoire au Québec.

Mots-clés: représentation communauté juive, curriculum, manuels scolaires, Histoire et éducation à la citoyenneté, Éthique et culture religieuse 


\section{The Representation of the Jewish Community in Quebec Textbooks}

\section{Abstract:}

This article examines the representation of the Jewish community and its history and culture in Quebec textbooks within two disciplines that are aimed at educating informed future citizens: History and Citizenship Education and Ethics and Religious Culture. Since most Quebec students encounter this community only through textbooks, it is important to establish the extent to which their education allows them to properly understand and develop positive attitudes toward it. Our study clearly shows that the Jewish community now enjoys greater visibility in textbooks, but this visibility does not always contribute to a better understanding of the community and its history in Quebec.

Key words: representation of the Jewish community, curriculum, textbooks, History and Citizenship Education, Ethics and Religious Culture

\section{Representación de la comunidad judía en los textos escolares de Quebec}

\section{Resumen:}

Este artículo estudia la representación de la comunidad judía, su historia y cultura en los textos escolares quebequenses de dos disciplinas escolares que tiene como objetivo la educación de futuros ciudadanos informados: Historia y Educación para la Ciudadanía y Ética y Cultura Religiosa. Dado que la mayoría de los estudiantes quebequenses se encuentran con esta comunidad sólo a través de los textos escolares, es importante determinar hasta qué punto la enseñanza que reciben les permite conocerla en profundidad 
y desarrollar actitudes positivas hacia ella. Este estudio pone de manifiesto que actualmente la comunidad goza de una mayor visibilidad en los textos escolares; sin embargo, dicha visibilidad no siempre contribuye a un mejor conocimiento de ella y de su historia en Quebec.

Palabras-clave: representación comunidad judía, currículum, texto escolar, Historia y Educación para la Ciudadanía, Ética y Cultura Religiosa

\section{Problématique et présentation de la recherche}

Implantée depuis plus de 250 ans au sein de la société québécoise, la communauté juive jouit d'indicateurs socio-économiques très favorables (Ministère de l'Immigration et des Communautés culturelles [MICC], 2005; Shahar et Karpman, 2004; Weinfeld, 2001). Elle a fortement contribué au développement du Québec, que ce soit à travers ses instances communautaires ou par l'apport de plusieurs de ses membres à la vie culturelle, sociale et politique (Anctil, 2006). Pourtant, elle reste encore largement méconnue par les autres Québécois, notamment les francophones en région (Jedwab, 2008; Sniderman, Northrup, Fletcher, Russel, et Tetlock, 1992). Le judaïsme est souvent assimilé aux pratiques hassidiques et la communauté dans son ensemble, à sa composante anglophone, pourtant de moins en moins importante ${ }^{1}$. La communauté a d'ailleurs été fortement ciblée lors du débat majeur sur «l'accommodement raisonnable» qui a secoué le Québec entre 2006 et 2008 (Mc Andrew, 2011; Potvin et Carr, 2008).

L’isolement, voire les tensions, qui ont prévalu jusqu'à la fin des années 1970, entre la communauté francophone et la communauté juive, surtout ashkénaze anglophone, explique en partie cet état de fait. Sans que l'antisémitisme ait été plus virulent au Québec, il y a pris des formes spécifiques liées à l'ambigüité du statut du groupe majoritaire ainsi qu'à l'influence de l'Église catholique (Bouchard, 2000; Delisle, 1992; Langlais et

\footnotetext{
${ }^{1}$ Aujourd'hui, $40 \%$ de la communauté sont francophones et $90 \%$ de ses membres sont bilingues (Shahar et Karpman, 2004).
} 
Rome, 1986; Weinfeld, 2008). Cependant, il semblerait qu'aujourd'hui la méconnaissance pourrait relever davantage du manque de contact généré par deux caractéristiques majeures de la communauté. D'une part, sa concentration métropolitaine et résidentielle reste encore marquée - selon le MICC (2005), 97,5\% des juifs québécois vivent à Montréal. D'autre part, une majorité de jeunes juifs fréquente des écoles de langue anglaise ou ethnoreligieuses trilingues. Cette situation résulte de facteurs historiques complexes. Au tournant $\mathrm{du} \mathrm{XX}^{\mathrm{e}}$ siècle, la communauté juive grandit rapidement avec l'arrivée de nombreux immigrants. Les écoles catholiques refusent alors d'accueillir ses enfants et les écoles protestantes, quant à elles, les acceptent as a matter of grace. La communauté juive préfère alors fonder ses propres écoles pour garantir la scolarisation de ses enfants (Corcos, 1997).

Puisqu'il y a si peu d'élèves juifs dans les écoles de langue française, surtout en région, une meilleure connaissance de la communauté peut être assurée essentiellement par le biais du curriculum formel ${ }^{2}$. Celui-ci peut en effet contribuer aussi bien à la reproduction qu'à la disparition de certains marqueurs culturels, linguistiques ou religieux par lesquels les communautés définissent leur identité et leur appartenance (Juteau, 2000), ainsi qu'au développement des attitudes réciproques et des compétences interculturelles des élèves (Banks, 2007; Mc Andrew, 2010). Mais c'est le matériel didactique, surtout lorsqu'il est soumis à un processus d'approbation par des instances gouvernementales (assuré au Québec par le Bureau d'approbation du matériel didactique, qui reflète l'intention du programme et donc l'image de la société contemporaine (Jadoulle, 2005) ou, du moins, «ce que les contemporains voudraient qu'elle soit» (Choppin, 1992, p. 163), en classe.

Des travaux menés ailleurs ont déjà illustré la présence de biais ethnocentriques dans le matériel didactique face au judaïsme et à la communauté juive. En Allemagne, par exemple, les manuels peinent à dépasser l'image des Juifs comme victimes. En accordant, depuis les années 1990, une place plus importante à l'histoire des Juifs pour mieux

\footnotetext{
${ }^{2}$ Ce terme renvoie au programme de formation de l'école québécoise ainsi qu'aux manuels approuvés par le Bureau d'approbation du matériel didactique (BAMD) au Ministère de l'Éducation, du Loisir et du Sport.
} 
l'intégrer dans l'histoire du pays, les manuels la classent souvent dans la rubrique de l'altérité (Lässig et Pohl, 2009). C'est aussi la conclusion principale qui ressort de l'étude d'Allouche-Benayoun (2008) sur la place accordée au judaïsme et aux Juifs dans les manuels scolaires français. Après la présentation du judaïsme dans l'antiquité, les élèves ne découvrent les Juifs que dans des moments de crises desquels ils sont clairement les victimes: l'Affaire Dreyfus, l'antisémitisme croissant des années 1930 et les années Vichy. Or, comme le précise l'auteure, la compréhension de ces mêmes événements est difficile en dehors de toute continuité historique.

Si dans un Québec qui prône l'interculturalisme, les manuels tentent de présenter la société à travers les valeurs qui lui sont associées, dont le rejet des préjugés et l'antiracisme (Lebrun, 2007, p. 1), la meilleure façon d'intégrer l'histoire des minorités reste un sujet de débat. D'ailleurs, le nouveau programme d'Histoire et éducation à la citoyenneté (HÉC) du Québec (MELS, 2004) est notamment critiqué pour «l'accent, présumé excessif, mis [entre autres] sur la pluralité culturelle» (Éthier, Lantheaume, Lefrançois, et Zanazanian, 2008) de la société québécoise. Il reste que d'importants efforts ont été menés pour assurer, entre autres, un enseignement de l'histoire ou des religions avec une approche plus inclusive. L'étude récente du traitement de l'islam et des musulmans dans les manuels scolaires québécois montre bien l'amélioration de l'image de cette communauté ostracisée dans le passé (Oueslati et Mc Andrew, 2011).

Ceci dit, malgré, d'une part, l'implantation ancienne de la communauté juive et, de l'autre, les nombreux débats dont elle fait l'objet, aucune recherche récente au Québec, comme au Canada, ne nous permet de nous prononcer à cet égard. Certains s'y réfèrent dans un contexte plus général, en étudiant par exemple la représentation du racisme dans les manuels scolaires de l'Ontario (Montgomery, 2005). D'autres se sont intéressés à une représentation plus spécifique du judaïsme dans le cadre de l'enseignement protestant au Canada (Polly, 1992).

Notre étude tente de combler cette lacune en s'intéressant à la représentation de la communauté juive du Québec, et notamment son vécu passé et présent, dans deux 
programmes particulièrement susceptibles de l'aborder: Histoire et éducation à la citoyenneté (HÉC) et Éthique et culture religieuse (ÉCR). D’une part, les objectifs de ces programmes, à savoir «préparer les élèves à participer de façon responsable, en tant que citoyens, à la délibération, aux choix de société et au vivre-ensemble [...]» (MELS, 2004, p. 1) et contribuer à la «reconnaissance de l'autre» (MELS, 2008, p. 2), offrent des occasions parfaites pour aborder la diversité culturelle québécoise. D'autre part, les thèmes qu'ils abordent concernent de près ou de loin cette communauté. Ainsi, le programme d'HÉC du secondaire aborde l'Holocauste dans le cadre du cycle consacré à l'histoire mondiale et les débuts de la communauté juive au Québec dans le cycle traitant de l'histoire du Québec et du Canada. Le programme d'ÉCR, dont l'un des mandats consiste spécifiquement à présenter les religions du Québec, discute quant à lui du judaïsme en général ainsi que de son vécu au Québec.

Nous présenterons ainsi une analyse des huit ensembles didactiques ${ }^{3}$ approuvés d'HÉC du $2^{\mathrm{e}}$ cycle du secondaire (quatre de $3^{\mathrm{e}}$ année et quatre de $4^{\mathrm{e}}$ année) et des six manuels approuvés d'ÉCR (de la $1^{\text {re }}$ année à la $5^{\mathrm{e}}$ du secondaire) ${ }^{4}$ qui s'y rattachent. Le corpus étudié est composé de 63 séquences, dont 23 images, de longs paragraphes de texte, des encadrés et quelques mentions mineures de la communauté dans des légendes ou des tableaux.

Notre grille d'analyse, qui s'inspire de l'étude récente du traitement de l'islam dans les manuels scolaires mentionnée plus haut (Oueslati et Mc Andrew, 2011), s'organise autour de deux niveaux de lecture. Dans un premier temps, une analyse quantitative s'intéresse à la comparaison du nombre de pages et d'images consacrées à chaque thème dans les manuels ainsi qu'à la fréquence des tendances identifiées dans l'analyse qualitative. Cependant, l'analyse a relevé peu d'indices significatifs, dû au nombre limité

\footnotetext{
${ }^{3}$ Les ensembles didactiques comportent les manuels d'élèves et des guides d'enseignement dans lesquels les auteurs ajoutent des commentaires, diverses indications pratiques et des situations d'apprentissage et d'évaluation (SAE) pour introduire la matière en classe. Nous nous référons cependant le plus souvent seulement aux manuels, car les guides d'enseignement n'ajoutent que rarement quelques commentaires au traitement de la communauté juive du Québec.

${ }^{4}$ Puisque nous nous intéressons au curriculum formel, nous avons étudié seulement les manuels approuvés par le BAMD au MELS. La liste complète de ces manuels est accessible sur le site du ministère http://www3.mels.gouv.qc.ca/bamd/menu.asp.
} 
des manuels étudiés et aux indications du programme généralement respectées par les auteurs des manuels. Une approche qualitative d'analyse de contenu nous semblait alors plus pertinente: au-delà de l'exactitude historique qui pouvait être vérifiée facilement, nous avons réfléchi aux choix que les manuels ont faits d'aborder ou d'omettre différents faits historiques dans leurs présentations de la communauté afin de cerner son image qui s'en dégage.

Néanmoins, quelques limites à cette étude doivent être considérées. Il importe, tout d'abord, de considérer la place restreinte de l'histoire de la communauté juive dans les manuels scolaires ainsi que le temps alloué à l'enseignement de l'HÉC ou de l'ÉCR au cours d'une année scolaire. Ensuite, il faut reconnaître qu'il n'est pas possible de consacrer à chaque communauté étudiée le temps nécessaire pour la connaître profondément. La longue histoire de la communauté juive au sein de la société québécoise est, en ce sens, un obstacle à sa présentation complète. Il est clair par ailleurs que quelques-unes de nos observations s'expliquent davantage par des «effets du programme». En effet, pour suivre le programme, les manuels sont obligés de présenter d'abord les différents thèmes dans l'ordre chronologique puis, dans un deuxième temps, dans une approche thématique.

C'est pour toutes ces raisons que nous ne cherchons pas à déterminer quelle serait l'image parfaite de la communauté dans le curriculum. Notre objectif est de vérifier si l'image qui se dégage de cette présentation reflète sa réalité, passée et présente, et permet de mieux la connaître. Précisons par ailleurs qu'à la suite de l'étude présentée ici, nous rencontrerons des enseignants d'histoire et d'éthique et culture religieuse afin d'apprendre sur leur usage des manuels et des extraits étudiés ici. 


\section{La communauté juive dans le programme de formation de l'école québécoise}

Le programme d'HÉC aborde explicitement la communauté juive à travers trois repères culturels. Ayant comme objectif «d'élargir [la] vision du monde [des étudiants] et leur compréhension des réalités sociales en leur offrant l'occasion de porter un regard sur des phénomènes sociaux ou des tendances culturelles significatives» (MELS, 2004, p. 33), ces repères tracent le chemin que prennent tous les manuels pour présenter les différents thèmes à étudier.

Le premier repère culturel abordant directement la communauté juive, Ezekiel Hart, apparaît dans le quatrième chapitre de la $3^{\mathrm{e}}$ année, «Revendications et luttes dans la colonie britannique». Reconnu pour sa contribution autant au développement économique de sa ville natale, Trois-Rivières, qu'à celui d'autres institutions québécoises (comme la Banque de Montréal), Hart est le premier Juif à être élu à la Chambre d'assemblée en 1808. Néanmoins, il est expulsé aussitôt après son élection sous prétexte qu'il ne peut pas porter serment comme un «bon chrétien», en jurant sur le Nouveau Testament. Réélu l'année suivante, il est de nouveau expulsé. Hart abandonne alors ses espoirs de devenir politicien, mais ses fils, s'alliant à Joseph Papineau et au Parti patriote, réussissent quelques années plus tard (en 1832) à faire reconnaître les droits citoyens des Juifs (Schoenfeld, 2010).

La Torah de la Congrégation Shearith Israël a été remise en cadeau par des Juifs de Londres à la première congrégation juive de Montréal fondée en 1768 à l'occasion de la construction de leur première synagogue en 1777, qui est d'ailleurs demeurée le seul lieu de culte juif de l'Amérique du Nord britannique pendant 80 ans et jusqu'à la construction d'une nouvelle synagogue en 1846 par des Juifs ashkénazes nouvellement arrivés (Linteau, Durocher, Robert, et Ricard, 1989b). Repère culturel du premier thème de la $4^{\mathrm{e}}$ année, «Population et peuplement», son contenu se réfère pourtant à la même période que celui du repère précédant qui décrit les origines de la communauté juive québécoise. Cependant, dans l'objectif de permettre aux élèves d'envisager une même réalité sous différents angles, le programme les aborde à des moments du cursus de l'élève très 
éloignés l'un de l'autre. Le rapprochement que les élèves peuvent réellement faire entre les deux événements devrait donc être examiné.

Un troisième repère culturel, Irving Layton, est suggéré dans le $3^{\mathrm{e}}$ thème de la $4^{\mathrm{e}}$ année, «Culture et mouvement de pensée». Ce poète d'origine juive est reconnu pour sa contribution importante à la scène culturelle montréalaise qui fleurit après la Seconde Guerre mondiale. Il peut donc devenir un angle d'entrée pour parler de la contribution culturelle de la communauté juive à la vie culturelle au Québec.

Dans le programme d'ÉCR, le seul repère culturel qui aborde explicitement la communauté juive du Québec est Abraham de Sola. Ce rabbin de la congrégation Shearith Israël commence à enseigner à l'Université McGill en 1847 dans la chaire d'études juives, alors que des étudiants juifs ne sont admis que par quota. Cependant, d'autres repères du même thème, «Le patrimoine religieux québécois», abordent des réalités qui concernent aussi la communauté juive du Québec, comme «Les institutions religieuses québécoises et leur influence sur la culture québécoise».

Les connaissances historiques liées à l'objet d'interprétation de ces deux programmes proposent d'autres occasions pour aborder la communauté juive québécoise. Les politiques d'immigration canadienne, abordées dans le $1^{\mathrm{er}}$ thème de la $4^{\mathrm{e}}$ année (Population et peuplement), incluent les vagues d'immigration qui ont façonné entre autres la communauté juive montréalaise, mais aussi le refus du gouvernement canadien d'accueillir des réfugiés juifs d'Europe à partir de la crise économique des années 1930 et jusqu'à la fin de la Deuxième Guerre mondiale. Les manifestations d'antisémitisme au Québec, qui ne sont pas abordées explicitement dans le programme, peuvent être appréhendées comme des «formes d'expression ou manifestations culturelles», notamment celles liées au fascisme ( $3^{\mathrm{e}}$ thème, $4^{\mathrm{e}}$ année, «Culture et mouvement de pensée»). Enfin, puisque différents aspects de la réalité contemporaine de la communauté juive font régulièrement les manchettes au Québec, nous avons aussi considéré les chapitres consacrés aux «Enjeux de la société québécoise» d'aujourd'hui $\left(2^{\mathrm{e}}\right.$ et $3^{\mathrm{e}}$ cycle). 
Tableau 1

La communauté juive selon les repères culturels

\begin{tabular}{|c|c|c|}
\hline Programme & Repère & Chapitre suggéré \\
\hline $\begin{array}{l}\text { Histoire, } \\
3^{\mathrm{e}} \text { année } \\
\text { Histoire, } \\
4^{\mathrm{e}} \text { année }\end{array}$ & $\begin{array}{l}\text { Ezekiel Hart } \\
\text { La Torah de la congrégation de } \\
\text { Shearith Israel } \\
\text { Irving Layton } \\
\text { Formes d'expression ou } \\
\text { manifestations culturelles liées } \\
\text { au fascisme }\end{array}$ & $\begin{array}{l}\text { Ch.4 «Revendications et luttes dans la } \\
\text { colonie britannique», repères culturels d'ici; } \\
\text { Ch.1 Population et peuplement, repères } \\
\text { culturels d'ici; } \\
\text { Ch. } 3 \text { Culture et mouvement de pensée, } \\
\text { repères culturels d'ici } \\
\text { Ch. } 3 \text { Culture et mouvement de pensée, } \\
\text { connaissances théoriques liées à l'objet } \\
\text { d'étude }\end{array}$ \\
\hline $\begin{array}{l}\text { ÉCR, } 1^{\text {er }} \text { cycle } \\
\text { secondaire }\end{array}$ & Abraham de Sola & Thème: Le patrimoine religieux québécois \\
\hline
\end{tabular}

Les repères culturels des programmes s'attachant clairement aux débuts de la présence de la communauté au Québec, ils ne permettent pas d'appréhender son parcours historique et son développement, pourtant bien intégrés à l'histoire de la société québécoise. Cependant, les manuels, disons-le d'emblée, vont au-delà des exigences en intégrant des exemples non prescrits par les programmes témoignant de la place qu'occupe la communauté dans la société québécoise. Dans la prochaine partie, nous examinerons cette présentation plus en détail.

\section{La communauté juive dans les manuels scolaires}

La présentation de la communauté juive varie peu entre les différents manuels étudiés ici (voir liste complète dans la bibliographie). Aussi, nous analysons l'image qui se dégage à travers les trois thèmes par lesquels elle est principalement abordée: l'arrivée des Juifs au Québec, leurs relations avec les autres Québécois et leur contribution sociale et culturelle à la société québécoise. Nous insisterons néanmoins sur les différences constatées entre les manuels lorsqu'elles nous paraissent significatives. 


\subsection{L'arrivée des Juifs au Québec}

Interdits de séjour en Nouvelle-France, où le droit d'immigration est réservé aux catholiques, les Juifs n'arrivent au Bas-Canada qu'après la Conquête britannique et une première congrégation, Shearith Israël, est fondée à Montréal en 1768. Deux repères culturels (sur trois suggérés par le programme), le livre de la Torah de cette congrégation et Ezekiel Hart, se réfèrent à cette période. Or, comme nous l'avons précisé plus haut, le lien entre les deux événements, enseignés avec une année de décalage, ne peut se faire aisément. D'ailleurs, le contexte dans lequel ils sont abordés est aussi différent: Ezekiel Hart s'inscrit dans l'histoire politique du Canada et le livre de la Torah dans celle de l'immigration au pays.

\subsubsection{Ezekiel Hart: un premier élu juif à la Chambre d'assemblée}

Les élèves rencontrent la communauté juive pour la première fois à travers l'histoire de l'élection d'Ezekiel Hart à la Chambre d'assemblée. Tous les manuels d'HÉC de $3^{\mathrm{e}}$ année ( $4^{\mathrm{e}}$ chapitre), mais aussi deux manuels d'ÉCR et un manuel d'histoire de $4^{\mathrm{e}}$ année, lui consacrent un encadré. Un court texte (deux paragraphes plus ou moins longs), accompagné d'une photo (la même dans tous les manuels), décrit l'homme juif prospère originaire de Trois-Rivières qui, élu à la Chambre d'assemblée en 1807, est tout de suite exclu parce qu'il est juif. Les manuels n'omettent pas de raconter la fin «heureuse» de l'histoire lorsqu'en 1831 le droit de siéger à la Chambre est accordé aux minorités.

Bien que plusieurs éléments de la présentation de cet épisode important dans l'histoire politique du Canada puissent être questionnés, notamment l'influence du capital financier de Hart (tous les députés de l'époque devaient être riches pour pouvoir s'aventurer en politique) et ses alliances avec les anglophones (limitées à la question du financement des 
prisons), ce sont les explications proposées par les manuels pour comprendre l'expulsion de Hart qui retiennent notre attention.

Trois manuels, un d'HÉC et deux d'ÉCR, adoptent la cause officielle de l'expulsion de Hart de la Chambre d'assemblée: sa judaïté. Une explication très cohérente lorsqu'elle est présentée dans les manuels d'ÉCR. En effet, à travers cette affaire qui «constitue un épisode important de notre histoire qui appartient à notre patrimoine religieux» (Réflexions, p. 45), ils invitent les élèves à considérer les difficultés rencontrées par des minorités religieuses au Québec dans le passé. Ils célèbrent aussi sa contribution à l'acceptation, en 1832, de la «loi qui accorde enfin aux Juifs tous les droits et privilèges dont jouissent les citoyens du Bas-Canada, y compris celui de siéger comme député» (Être en société, p. 100).

Or, lorsque Fresques, un manuel d'HÉC, propose que «justement parce qu'il est de religion juive, la Chambre d'assemblée lui refuse le droit de siéger» (p. 12), cette explication nous semble plutôt réductrice. Le contexte politique de l'époque est complexe: Hart se fait élire au moment où la «querelle de prisons» divise la Chambre entre le Parti bureaucratique (britannique) et le parti patriote (français) autour de la question des frais de construction de nouvelles prisons. Hart, candidat indépendant, promet son soutien au Parti bureaucratique. Les francophones n'hésitent alors pas à utiliser sa judaïté comme prétexte pour écarter leurs adversaires (Weinfeld, 2001). En discutant de l'usage cynique que l'on peut faire des différences culturelles, religieuses ou sociales dans le milieu politique, l'affaire Hart s'intègre dans un enseignement plus général sur la démocratie et les droits civiques au Québec et sur l'histoire politique de la confédération. La complexité d'un fait historique et de son analyse est aussi à signaler. Le fait qu' «en 1831, le Bas-Canada est le premier territoire de l'Empire britannique à accorder tous les droits politiques aux Juifs»» (Repères, p. 239), par lequel tous les manuels terminent leur présentation, prend alors toute sa signification.

Mais l'explication politique peut aussi amener à un renforcement de préjugés lorsque, comme le fait le manuel Le Québec: une histoire à construire, l’on explique que, «comme 
les Juifs du Canada sont plus proches du parti anglais, les députés francophones l'écartent de la Chambre en prétextant que les Juifs n'ont pas le droit de se présenter en politique» (p. 468). Cette généralisation s'attache aux préjugés en même temps qu'elle les nourrit, négligeant de préciser que Hart, candidat indépendant, est élu en dépit des candidatures posées par deux francophones (majoritaires dans la conscription de Trois-Rivières) et un anglophone.

Paradoxalement, ce dernier manuel est aussi le seul à aborder clairement l'antisémitisme dans ce contexte, sans pour autant inviter clairement les élèves à réfléchir aux dérives dangereuses d'une rivalité politique. Il questionne les élèves sur «comment s'appelle le sentiment d'hostilité envers les Juifs?» et «ce sentiment existe-t-il toujours de nos jours?» (p. 275). Cependant, il ne propose aucun élément de réponse pour les aider à aborder ce sujet difficile. D'ailleurs, aucun manuel n'évoque la communauté juive de l'époque, que ce soit pour mieux comprendre l'affaire ou considérer son influence sur la communauté elle-même. La communauté juive d'aujourd'hui n'est pas non plus mentionnée.

\subsubsection{La Torah comme symbole de la communauté}

Cadeau fait par des Juifs de Londres à la congrégation Shearith Israël à l'occasion de l'inauguration de la première synagogue de Montréal, la Torah de la congrégation Shearith Israël est le repère qui permet d'aborder les débuts de la communauté du Québec. Trois manuels d'histoire de $4^{\mathrm{e}}$ année consacrent en effet un encadré avec photo à cet

artefact religieux $\left(1^{\mathrm{er}}\right.$ thème, «Population et peuplement»), mais un quatrième fait fi de cette suggestion, s'attachant plutôt à l'histoire de la communauté en général. Il accompagne d'ailleurs sa présentation avec la photo d'une autre synagogue.

Les manuels expliquent alors que «l'immigration juive ne débute véritablement qu'avec la Conquête de 1760» (Repères, p. 40). Ils insistent sur les affinités des Juifs avec les anglophones, notamment avec l'armée britannique qu'ils fournissent «en vivres et en 
munitions» (Québec: une histoire à construire, p. 44) et décrivent le «climat favorable à l'expansion de la communauté juive» (Repères, p. 40) avec l'arrivée des Loyalistes. Par contre, le fait que «les Juifs n'étaient pas les bienvenus en Nouvelle-France» (Fresques, p. 46) n'est rapporté que dans un manuel qui permet de porter un regard plus complexe sur les affinités qui s'établissent entre les Juifs et les anglophones.

Les encadrés décrivent ensuite la construction de la synagogue et expliquent l'importance du livre de la Torah, s'intéressant davantage à une pratique religieuse qu'à la réalité de la communauté juive de l'époque ou l'influence de cette immigration sur la société québécoise d'alors. Cet attachement aux aspects religieux plutôt qu'aux aspects sociaux dans un manuel d'histoire nous semble questionnable, d'autant plus en comparant ce traitement avec celui que proposent les deux manuels d'ÉCR (Tête-à-tête et Être en société), évoquant plutôt la diversité des pratiques (notamment entre les traditions sépharade et ashkénaze) et en montrant les différents sites qui abritaient cette synagogue au fil des ans. Leur approche permet en effet d'actualiser la présence juive à Montréal et de dépasser la simple histoire d'un artefact religieux.

Les manuels d'ÉCR expliquent aussi davantage l'interdiction aux Juifs de s'établir en Nouvelle-France. L'un d'eux (Tête-à-tête, p. 177) rapporte même l'histoire de la jeune Esther Brandeau qui émigre au Québec déguisée en garçon catholique, pour être repérée par les autorités et renvoyée en France par le roi l'année suivant son arrivée. Cette anecdote permet de mieux appréhender le contexte politique de l'époque et de considérer différemment le faible pourcentage de Juifs francophones vivant au Québec jusqu'à la deuxième moitié du $\mathrm{XX}^{\mathrm{e}}$ siècle, lorsque des Juifs francophones arrivent principalement d'Afrique du Nord. Elle invite en même temps à considérer d'autres manifestations de ce clivage religieux, tel le refus des catholiques d'accueillir dans leurs écoles les Juifs (qui se retrouvent ainsi dans les écoles anglophones). 


\subsubsection{Immigration juive au Québec}

Les trois manuels d'HÉC de $4^{\mathrm{e}}$ année se réfèrent à l'exemple de la communauté juive en discutant l'immigration au Québec au tournant du siècle. La croissance rapide de la communauté grâce aux vagues d'immigration successives (de 7607 individus en 1901 60087 en 1931), notamment des Juifs arrivant d'Europe de l'Est où ils sont régulièrement victimes de persécutions, marque en effet l'histoire du Québec (Linteau, et al., 1989b). Il n'est pas étonnant que les manuels s'y réfèrent, comme d'ailleurs à l'histoire de la communauté italienne, pour témoigner ainsi d'une période d'immigration importante au Québec et même au Canada. La place qu'accordent les manuels à la communauté juive dans ce contexte est pourtant inégale.

L'un des manuels se contente de montrer la croissance des communautés ethniques, dont celle juive, à travers des diagrammes et explique que, par leur «tendance à se regrouper entre elles» (Fresques, p. 78), ces communautés influencent le paysage physique et culturel de Montréal. Les deux autres manuels consacrent une courte section à la communauté juive et une autre à la communauté italienne. Ils s'arrêtent également sur les statistiques et précisent qu'en 1930, les Juifs représentent «le groupe ethnique et culturel le plus important du Québec après les Canadiens français et les personnes de descendance britannique» (Présences, p. 52). Cependant, ils expliquent aussi les motivations des Juifs, d'abord celle de fuir «les persécutions dont ils sont victimes en Russie et en Europe de l'Est» (Le Québec: une histoire à construire, p. 64), puis celle de s'installer à Montréal, «où se développe une communauté structurée qui vient en aide aux nouveaux arrivants» (Présences, p. 52). Leur grand effort pour s'intégrer économiquement est aussi souligné.

La contribution des immigrants à la richesse de la culture québécoise est aussi clairement célébrée dans les manuels d'HÉC qui présentent le boulevard St-Laurent, repère culturel du thème Population et peuplement. Les Juifs sont mentionnés parmi d'autres communautés qui s'y installent au début du $\mathrm{XX}^{\mathrm{e}}$ siècle, transformant le paysage de la ville. 
Les effets que pouvait avoir une immigration aussi importante sur la communauté existante ne sont, par contre, pas mentionnés, bien qu'ils représentent un aspect important de ce phénomène historique qui est l'immigration. Ni les diverses institutions dont se dote la communauté juive pour organiser sa vie culturelle, sociale et économique, ainsi que ses relations avec les autres Québécois (le Congrès juif canadien), ni la division de la communauté, pour la première fois, en plusieurs courants idéologiques, religieux et politiques (Weinfeld, 1993, 2001), ne sont jamais abordées dans les manuels d'HÉC. Pourtant, comme l'explique le manuel d'ÉCR Tête-à-tête, «la diversité religieuse, sociale et économique qui caractérise les Juifs à Montréal et ailleurs est telle qu'il existe en fait plusieurs communautés juives plutôt qu'une seule» (p. 177). C'est ainsi le seul manuel à s'intéresser aussi à l'identité laïque du Juif non religieux et à son rôle dans le développement d'un judaïsme culturel.

Ne proposant aucun lien explicite entre la réalité historique étudiée et la communauté actuelle, les manuels d'HÉC laissent les élèves avec une image partielle qui ne décrit plus la réalité contemporaine. En présentant par exemple des statistiques qui n'atteignent presque jamais les années 2000, ils proposent de conserver le cliché d'un passé lointain. La description de la communauté juive comme étant la minorité ethnique la plus importante au Québec ne correspond pas non plus à la réalité contemporaine, mais renforce les idées reçues à son égard. D’ailleurs, les manuels ne suggèrent aucun lien explicite entre les deux repères culturels exposés, qui décrivent pourtant une réalité historique un peu plus complexe que l'arrêt sur image d'une communauté dans un moment de l'histoire.

\subsection{Différentes facettes des relations de la communauté juive avec les autres Québécois}

Malgré leur présence ancienne et leur intégration linguistique, économique et sociale poussée (Anctil, 2009), les contacts entre les Juifs et les autres Québécois ne sont pas toujours harmonieux. Des facteurs complexes, historiques comme actuels, peuvent 
expliquer cet état de fait. D'une part, l'imaginaire de la communauté juive est teinté par des manifestations d'antisémitisme touchant le Québec des années 1930 (Tulchinsky, 1993; Weinfeld, 2001) et le refus canadien d'accueillir des réfugiés juifs qui fuient l'Europe à la veille de la Deuxième Guerre mondiale (dont la devise canadienne none is too many résume parfaitement la politique) est aussi significatif (Linteau, Durocher, Robert et Ricard, 1989a). Plus récemment, des tensions politiques autour de deux référendums (1982 et 1995), mais aussi de la loi 101 et ses diverses annexes (notamment dans les écoles) ont ébranlé la place qui semblait acquise par la communauté juive. D'autre part, la fréquentation par une majorité (même si faible) d'élèves juifs (Shahar et Karpman, 2006) des écoles juives, fondées au début des années 1900 suite au refus de les accepter dans les écoles catholiques, et leur seule «tolérance» dans les écoles protestantes, les éloignent de la majorité des Québécois.

Les débats récents (2008) autour des «accommodements raisonnables» ont ressuscité les tensions et le sentiment d'incompréhension permanente. Ils ont surtout laissé l'impression que, à bien des égards, la communauté juive continue d'être perçue comme étrangère à la société québécoise. Il nous paraissait donc essentiel de considérer la manière dont les manuels discutent des différentes facettes des relations entre la communauté juive et les autres Québécois, explicitement comme implicitement.

\subsubsection{Des manifestations antisémites au Québec}

L’antisémitisme n'est pas explicitement abordé par les deux programmes étudiés ici. Pourtant, trois manuels d'HÉC et deux d'ÉCR l'évoquent clairement. L'«antisémitisme québécois», comme l'explique Présences, «ne se manifeste pas par la violence, mais plusieurs Québécois dénoncent la présence des Juifs et soutiennent qu'ils refusent de s'assimiler» (vol. 1, p. 53). Plus spécifiquement, «un rare exemple d'antisémitisme du Québec», celui d'«une affiche antisémite» qui accueille les visiteurs au village Sainte-Agathe dans les années 1940, est présenté dans le volume suivant. Un autre manuel présente cette affiche à côté d'«un avis antinazi [qui] invite la population à une rencontre 
contre le nazisme et Hitler» en avril 1939 (Fresques, p. 81), invitant ainsi les élèves à considérer la complexité du phénomène.

Ce dernier manuel est aussi le seul à présenter un autre incident marquant de l'histoire de la communauté juive à Montréal: la grève d'internes de l'hôpital Notre-Dame qui, en 1934, protestent contre le recrutement d'un interne juif, obligeant ainsi ce dernier à renoncer à son stage. Le manuel rapporte un extrait de l'entrevue que le jeune médecin juif a accordée au journal Le Devoir le 19 juin 1934 dans lequel il explique qu'il ne pouvait que démissionner s'il considérait les intérêts de «la race juive», des patients de l'hôpital Notre-Dame et de sa direction qui l'a par ailleurs soutenu tout au long du conflit (p. 81). Ce manuel offre ainsi la seule occasion à la communauté juive de «se faire entendre» par sa propre voix et les conséquences personnelles de l'antisémitisme, qui ne peut plus être considéré comme de seules tensions intercommunautaires, se dévoilent.

Ce manuel évoque à nouveau l'antisémitisme lorsqu'il présente l'arrêt de l'immigration dans le contexte de la crise financière des années 1930. Tout comme Le Québec: une histoire à construire, il discute du refus du gouvernement canadien d'accueillir des Juifs qui demandent l'asile au Canada (le SS St. Louis) et de l'ambiance antisémite qui se répand dans le pays comme au Québec. Les deux manuels profitent d'ailleurs de l'occasion pour définir l'antisémitisme dans le glossaire comme un «sentiment d'hostilité» ou du «racisme» dirigé contre les Juifs. Or, en laissant croire, à tort, que le refus d'accueillir les Juifs était directement lié à la guerre, les manuels manquent l'occasion de discuter de la complexité d'une décision politique influencée notamment par les craintes des syndicats d'ouvriers appauvris par la crise économique et des préjugés généraux à l'égard des réfugiés.

Des manifestations antisémites plus récentes sont discutées dans trois manuels. Le manuel d'HÉC Présences ( $3^{\mathrm{e}}$ année), propose de discuter des tensions et difficultés que peut rencontrer une «société d'accueil» (p. 252) en montrant des images actuelles telles «des femmes musulmanes à Hérouxville» ou les graffitis de jeunes Québécois d'origine haïtienne en «quête d'identité». Une des photos, intitulée «Val-David: des camps d'été 
brûlent» (p. 253), montre un homme juif hassidique qui parle au téléphone cellulaire devant les restes d'une maison brûlée. Une légende décrit brièvement l'incident en question et explique entre autres que le mouvement hassidique juif «rejette la modernité technique» (en glossaire, p. 253).

Cette description nous pose plusieurs problèmes. Tout d'abord, en associant clairement la communauté juive implantée depuis plus de deux siècles au Québec à d'autres, récemment arrivées, elle ne permet pas aux élèves d'appréhender la signification du rejet de ces membres. En effet, après 250 ans de présence au Québec, on aurait pu croire que ces tensions seraient amoindries. Ensuite, en montrant la photo d'un homme hassidique comme représentant ces tensions, le manuel renforce des préjugés existant déjà à l'égard de la communauté. La définition erronée du terme «hassidique» en renforce cette stigmatisation: en effet, les hassidim utilisent régulièrement la technologie pour faciliter leur quotidien régulé par de nombreuses prescriptions religieuses ${ }^{5}$. Rappelons-le: la photo elle-même montre le porte-parole de ce groupe religieux en train d'utiliser son téléphone cellulaire! La modernité à laquelle s'opposent les hassidim est philosophique et invite à adapter la pratique religieuse à la vie contemporaine (en se rendant, par exemple, en voiture à la synagogue). Le manuel, qui n'aborde d'ailleurs pas la notion d'antisémite à cette occasion, semble s'attacher par ce portrait au même préjugé qui est aux fondements de telles manifestations.

L'antisémitisme contemporain est aussi discuté dans le manuel d'ÉCR Tête-à-tête, qui s'arrête notamment sur les réactions possibles de la société face à cette attitude. C'est ainsi que, en étudiant «les limites de la liberté» ( $1^{\mathrm{er}}$ thème en éthique), la loi canadienne contre l'expression haineuse, «invoquée à l'encontre d'individus ayant proféré des propos antisémites» (p. 39-41), est expliquée et un exemple précis de son application est rapporté. La définition du terme «antisémitisme» dans le glossaire insiste par ailleurs sur les spécificités de ce type de racisme. Dans un autre volume ( $4^{\mathrm{e}}$ secondaire), ce manuel consacre une page à un incendie criminel «qui a dévasté [une] bibliothèque [dans une]

${ }^{5}$ Les exemples sont nombreux. Des minuteries de Shabbat qui éteignent les lumières le soir et les rallument le matin leur permettent de profiter de l'électricité pendant la journée sans en gaspiller la nuit. Les plaques électriques permettent de garder la nourriture chaude sans garder le four allumé. 
école primaire juive [et] a suscité une dénonciation aussi ferme qu'immédiate de la communauté juive comme de tous les paliers politiques» (p. 14). En rapportant les propos du groupe Loco Locass, qui décide de participer à une «manifestation de la société civile artistique», ainsi que ceux du politicien Amir Khadir, le manuel invite les élèves à réfléchir aux divers enjeux sociaux, politiques et culturels que soulèvent ces manifestations contemporaines d'antisémitisme.

Ces deux exemples montrent l'intérêt de parler de ces phénomènes sans oublier leur complexité et leur étendue sociale. Ils montrent aussi l'indignation générale de telles manifestations de racisme et de haine. Enfin, ils proposent une autre lecture de l'histoire de la communauté juive du Québec qui reste marquée par ces incidents ainsi qu'une clé de compréhension de la forte solidarité qui la qualifie encore aujourd'hui (Tulchinsky, 1993; Weinfeld, 2001).

C'est pourquoi il nous semble aussi intéressant de signaler l'absence de toute considération de la place qu'avait la communauté juive dans le débat autour des «accommodements raisonnables» qui a secoué le Québec en 2008. Les manuels proposent comme seuls exemples les questions autour du port du voile islamique ou encore du kirpan sikh, mais ne s'interrogent jamais sur les tensions qu'ont provoquées les pratiques juives. De la même manière, le manuel Présences ( $4^{\mathrm{e}}$ année), qui reprend les résultats d'un sondage de l'Association d'études canadiennes (2003), discute de l'attitude des Québécois et des Canadiens à l'égard des minorités ethniques (p. 154). Il s'arrête sur leurs mauvaises perceptions des Autochtones et des Arabes, mais ne s'intéresse pas aux données concernant les Juifs, qui montrent pourtant qu'ils sont les seuls à être moins bien perçus tant par les Québécois que par les Canadiens. Ce constat reste donc sans aucun commentaire.

\subsubsection{Le mouvement fasciste québécois et les Juifs}

Le mouvement fasciste québécois fait partie des connaissances historiques que le programme d'histoire propose d'explorer. Avec sa doctrine antisémite et sa participation malgré tout significative à la vie politique québécoise des années 1930, il nous paraissait 
intéressant d'examiner les extraits que lui consacrent les manuels. Insistant notamment sur son affiliation au fascisme européen en général et au nazisme en particulier, les manuels expliquent surtout le rôle que cette idéologie jouait dans l'avènement de la Deuxième Guerre mondiale et dans «l'extermination des Juifs européens» (Présences, p. 56). La photo des objets décorés par la croix gammée qu'a retrouvés la police dans les locaux du mouvement à Montréal, qui accompagne tous ces extraits, confirme l'adhésion des militants québécois à cette idéologie.

Cependant, seulement deux manuels évoquent l'influence que cette idéologie avait sur le paysage politique québécois, notamment en précisant son attrait sur certains milieux intellectuels au Québec. Fresques ( $4^{\mathrm{e}}$ année) parle de l'écho que l'on trouve dans les écrits de «quelques nationalistes canadiens-français» (p. 63) et Repères ( $3^{\mathrm{e}}$ année), explique que «certaines élites québécoises proches du clergé catholique vouent une sympathie envers les régimes fascistes ou autoritaires qui se sont installés en [Europe]» (p. 389). Ils insistent aussi, à juste titre, sur le fait que les Québécois n'ont porté de manière générale que peu d'intérêt au parti fasciste, qui restait donc marginal dans le paysage politique québécois comme canadien.

L'explication que propose le troisième manuel, Le Québec: une histoire à construire, concernant l'intérêt que suscite ce mouvement parmi de jeunes Québécois est plus problématique. Selon ce manuel, «le mouvement s'attaque particulièrement aux Juifs et à leurs tendances socialistes» (p. 400), présentées déjà dans les deux pages précédentes consacrées aux «promoteurs du socialisme», les Juifs. Ces derniers sont alors décrits comme «anticonformistes [qui] s'opposent au modèle capitaliste établi et perçoivent le socialisme comme un moyen de renverser les anciennes normes et traditions» (p. 398). À travers cette généralisation, à laquelle le manuel consacre une place considérable (en comparaison avec la place accordée au socialisme dans d'autres manuels et avec celle d'autres mouvements politiques dans ce même manuel), le manuel justifie implicitement les inquiétudes ressenties à l'époque par les militants fascistes. Pire encore, il reproduit clairement un des préjugés à l'égard des Juifs: leur désir de renverser l'ordre politique et de contrôler le monde à leur manière. 


\subsubsection{La contribution de la communauté juive à la société québécoise}

La communauté juive s'intègre dans la société québécoise notamment à travers ses contributions à la scène culturelle. Les manuels en parlent à plusieurs occasions. Ils abordent la grande influence des immigrants, y compris des Juifs, sur l'urbanisation de Montréal à travers l'histoire du boulevard St-Laurent, un repère culturel du programme, et intègrent différents aspects culturels dans les descriptions de la communauté juive ellemême. Ainsi, la gastronomie juive attire l'attention des trois manuels d'histoire de $4^{\mathrm{e}}$ année alors que le yiddish, la langue parlée par les immigrants juifs d'Europe de l'Est, est mentionné dans l'un d'entre eux (Le Québec: une histoire à construire).

C'est pourtant en faisant référence à quelques célébrités juives que les manuels évoquent le plus souvent cette contribution. Ils nomment Leonard Cohen, un chanteur montréalais de renommée internationale, Irving Layton, un poète d'origine juive (repère culturel du programme), ou encore Mordecai Richler, considéré, selon un des manuels, «comme l'un des plus grands auteurs anglophones du Canada» (Présences, p. 53).

Une autre présentation de ce même personnage excentrique semble contribuer plutôt à renforcer l'image d'une communauté distante et distincte. L'auteur, explique le manuel Repères, «[...] est très peu lu par les francophones, même si ces ouvrages ont été traduits en français. Ses interventions provocatrices sur la question nationale et dans les débats linguistiques au Québec ont parfois fait oublier son talent d'écrivain» (p. 227). Se référant sans doute au tollé général qu'a provoqué le livre Oh Canada! Oh Québec! Requiem pour un pays divisé (Richler, 1992), le manuel ne prend pas le temps de rappeler l'indignation que les propos publics de l'auteur ont aussi soulevée au sein de la communauté juive. Intégrée par ailleurs dans une section consacrée à «l'apport des anglophones» (p. 227), cette présentation de l'auteur renforce aussi l'image d'une communauté juive indissociable de la population anglophone.

L'image qui se reflète de cet échantillon malgré tout limité est celle d'une communauté culturelle distincte, distante et étrangère. Aucun manuel d'histoire ne rappelle la contribution de diverses institutions fondées par la communauté pour soutenir 
la culture et la création artistique en général et aucune célébrité juive francophone n'est nommée. Ils se bornent tous à dresser le portrait d'une communauté qui fait avant tout office d'altérité au sein de la société québécoise.

Pourtant, la présence marquée de cette communauté au centre-ville de Montréal et ses nombreuses initiatives culturelles, comme la fondation de la bibliothèque juive et le développement d'un théâtre yiddish, ont certainement marqué le paysage culturel montréalais de l'époque. L'absence de toute référence aux écoles juives qui font pourtant souvent les manchettes des journaux québécois reste sans doute l'exemple le plus flagrant. Plutôt que le traiter comme un sujet conflictuel, les manuels d'HÉC auraient pu expliquer le contexte historique qui amène la communauté juive à inaugurer un système d'éducation indépendant.

La comparaison avec le manuel d'ÉCR Tête-à-tête montre une autre approche. Ce manuel accorde une place importante aussi bien aux célèbres artistes et scientifiques juifs du Canada et d'ailleurs, qu'aux personnalités juives québécoises, dont l'architecte Safdie, créateur d'Habitat 67 et du Musée des beaux-arts, le chimiste Polanyi et l'auteur francophone Naïm Katan, en plus de Mordecai Richler et Leonard Cohen. Il mentionne aussi la contribution de nombreux organismes juifs au monde culturel québécois, sans oublier les efforts de la communauté à maintenir sa propre culture vivante, notamment à travers le théâtre yiddish. Aussi, même cet aspect plutôt historique retrouve un lien avec l'actualité culturelle québécoise.

\section{Conclusion}

La communauté juive a fait une entrée assez significative dans les nouveaux manuels scolaires étudiés ici, du moins si l'on considère qu'elle n'est jamais clairement mentionnée dans le programme d'histoire précédent. Grâce aux repères culturels du nouveau programme, qui proposent des portes d'entrée différentes en matière de connaissances historiques, la communauté juive, son histoire et même sa culture, jouissent d'une visibilité beaucoup plus évidente. Les manuels, qui ne se limitent d'ailleurs pas à ces 
suggestions, se réfèrent aussi à la communauté juive pour témoigner des moments fondateurs de la société québécoise.

Ces acquis sont particulièrement marqués à deux niveaux. Le premier concerne les débuts de la communauté au Québec, c'est-à-dire l'arrivée des Juifs après la Conquête ainsi que les vagues importantes d'immigration qui font de cette minorité ethnique la plus importante au pays. Le deuxième aspect que les manuels traitent clairement, et ce sans que le programme ne l'aborde d'aucune manière, est l'antisémitisme au Québec dans le passé.

Cependant, l'image de la communauté telle qu'elle se dégage de sa présentation dans les manuels souffre de trois limites principales. La première réside dans son historicité. Les manuels s'attachent uniquement aux faits historiques sans jamais les rattacher à la communauté qui habite encore aujourd'hui au Québec et au Canada. Ils accentuent ainsi les «effets du programme» dont nous avons parlé plus tôt: la référence aux moments forts pour démontrer une réalité historique complexe, sans s'arrêter sur son contexte plus général. Si cette approche enrichit l'enseignement de l'histoire en général, elle laisse facilement les élèves avec des arrêts sur image, de vrais clichés sur la communauté juive. Les différents encadrés deviennent alors des clins d'œil folkloriques dans un cours d'histoire et leur utilité ne dépend que de l'usage qu'en font les enseignants.

Le portrait de la communauté que tracent les manuels est celui d'une communauté immigrante, religieuse et anglophone. Un portrait qui est aujourd'hui plus que partiel. En effet, les manuels, qui ne s'attardent pas sur l'immigration juive arrivée après la Deuxième Guerre mondiale, n'évoquent pas la diversité religieuse (des laïcs aux ultra-orthodoxes), culturelle (sépharades et ashkénazes), ethnique (de tous les pays du monde) et linguistique (de plus en plus francophone) de cette communauté.

Une deuxième limite réside dans le fait que, même si on y fait parfois référence, les aspects sociaux de la communauté sont le plus souvent passés sous silence. Aussi, son apport culturel et social non religieux, pourtant majeur au sein même de la communauté (seulement $12 \%$ sont ultra-orthodoxes) est à peu près absent. 
La comparaison avec les manuels d'ÉCR est plus qu'éclairante à cet égard. Afin de répondre à l'objectif de préparer les élèves à un meilleur «vivre ensemble», les manuels discutent de la complexité et de la diversité de cette communauté, notamment lorsqu'ils évoquent clairement le pluralisme ethnique, bilingue et religieux en son sein et la variété des pratiques religieuses. La description du contexte contemporain, notamment en ce qui concerne la contribution de la communauté à la vie culturelle de la société québécoise et les manifestations d'antisémitisme, doit aussi être signalée.

Ce qui nous amène à la troisième limite de ce traitement. Si notre étude illustre l'apport des manuels en matière d'éducation interculturelle en ce qui concerne le traitement de l'histoire, elle démontre aussi que ces derniers réussissent moins bien à répondre aux objectifs du programme quant à l'éducation à la citoyenneté, à savoir ceux d'aider les élèves à mieux comprendre la société dans laquelle ils vivent et de les préparer à y agir en tant que citoyens. En effet, non seulement la communauté d'aujourd'hui n'est pas décrite, mais son portrait dans les manuels justifie presque les craintes des autres Québécois face à ses membres.

Le manque de contextualisation dont notre étude fait état représente donc le biais principal dans le traitement de la communauté juive dans les manuels. C'est pourquoi nous avons jugé essentiel de considérer, dans un deuxième temps, l'usage qu'en font les enseignants en classe, interrogeant également leur perception de ce traitement: pourront-ils combler les lacunes de ce traitement? Cette analyse fera l'objet d'une publication future.

Note: Ce texte est issu d'un rapport publié par la Chaire de recherche du Canada sur l'Éducation et les rapports ethniques, au CEETUM, Université de Montréal, en janvier 2011. Pour le rapport complet, consultez le site internet de la Chaire http://www.ceetum.umontreal.ca/. 


\section{Références}

Allouche-Benayoun, J. (2008). Juifs et judaïsme dans les manuels d'Histoire des lycées en France. In B. Lefebvre et S. Trigano (éd.), L’Image des Juifs dans l'enseignement scolaire (p. 39-124). Paris: Éditions du Nadir.

Anctil, P. (2006). Les communautés juives de Montréal. In M.-C. Rocher et M. Pelchat (éd.), Le patrimoine des minorités religieuses au Québec. Richesse et vulnérabilité (p. 37-60). Québec: PUL.

Anctil, P. (2009). Un patrimoine en mouvance: l'apport decisive des communautés non chrétiennes. In S. Lefebvre (éd.), Le patrimoine religieux du Québec. Éducation et transmission du sens (p. 67-85). Québec: Les Presses de l'Université Laval.

Banks, J.A. (2007). Educating citizens in a multicultural society (2e éd.). New York: Teachers College Press.

Bouchard, G. (2000). Les rapports avec la communauté juive: un test pour la nation québécoise. In P. Anctil, Robinson, Ira et G. Bouchard (éd.), Juifs et Canadiens français dans la société québécoise (p. 13-31). Québec: Septentrion.

Choppin, A.L. (1992). Les manuels scolaires: histoire et actualité. Paris Hachette.

Corcos, A. (1997). Montréal, les juifs et l'école. Québec: Septentrion.

Delisle, E. (1992). Le traître et le juif: Lionel Groulx, le Devoir et le délire du nationalisme d'extrême droite dans la Province de Québec, 1929-1939. Montréal, Outremont: Étincelle.

Éthier, M.-A., Lantheaume, F., Lefrançois, D. et Zanazanian, P. (2008). L'enseignement au Québec et en France des questions controversées en histoire: tensions entre politique du passé et politique de la reconnaissance dans les curricula. Éducation et Francophonie. Rapport ethniques et éducation: perspectives nationales et internationales, 36(1), 65-85.

Jadoulle, J.-L. (éd.). (2005). Les manuels scolaires d'histoire: Passé, présent, avenir. Louvain-la-Neuve: UCL Université catholique de Louvain.

Jedwab, J. (2008). Attitudes Towards Jews and Muslims: Comparing Canada with the United States and Europe. Montréal: Association d'études canadiennes.

Juteau, D. (2000). L'ethnicité et ses frontières. Montréal: Presses de l'Université de Montréal.

Langlais, J., et Rome, D. (1986). Juifs et Québécois français: 200 ans d'histoire commune. Montréal: Fides. 
Lässig, S. et Pohl, K.H. (2009). History Textbooks and Historical Scholarship. History Workshop Journal, 67(1), 125-139.

Lebrun, M. (2007). La figure de l'étranger dans les manuels québécois de français langue maternelle. In M. Lebrun, P. Aubin, M. Allard et A. Landry (Eds.), Le manuel scolaire d'ici et d'ailleurs, d'hier à demain (p. 1-31). Québec: Presses de l'Université du Québec.

Linteau, P.-A., Durocher, R., Robert, J.-C. et Ricard, F. (1989a). Histoire du Québec contermporain. Le Québec depuis 1930 (Vol. 2). Montréal: Boréal.

Linteau, P.-A., Durocher, R., Robert, J.-C. et Ricard, F. (1989b). Histoire du Québec contermporain. De la Confédération à la crise (1867-1929) (2 ${ }^{\mathrm{e}}$ éd. Vol. 1). Montréal: Boréal.

Mc Andrew, M. (2010). Les majorités fragiles peuvent-elles s'ouvrir au pluralisme? Enjeux éducatifs au Québec et en Europe. Montréal: Presses de l'Université de Montréal.

Mc Andrew, M. (2011). La controverse sur 1'accommodement raisonnable au Québec: atout ou obstacle au rapprochement interculturel? In A. Goldschlager et R. Klein (éd.), L'époque du St. Louis: se rappeler le passé, aller de l'avant. Publié par le Groupe de travail national sur l'éducation, la mémoire et l'étude de l'Holocauste. Documents téléaccessibles à l'adresse: www.stlouis2009conference.ca et http://www.stlouis2009conference.ca. Toronto Groupe de travail national sur l'éducation, la mémoire et l'étude de l'Holocauste.

Ministère de l'Éducation, du Loisir et du Sport (2004). Programme de formation de l'école québécoise: Histoire et éducation à la citoyenneté. Domaine de l'univers social.

Ministère de l'Éducation, du Loisir et du Sport (2008). Programme de formation de l'école québécoise: Éthique et culture religieuse.

Ministère de l'Immigration et des Communautés culturelles (2005). Portrait statistique de la population d'origine ethnique juive, recensée au Québec en 2001. Québec: Ministère de l'Immigration et des Communautés culturelles.

Montgomery, K. (2005). Imagining the Antiracist State: Representations of racism in Canadian history textbooks. Discourse: Studies in the Cultural Politics of Education, 26(4), 427-442.

Oueslati, B., et Mc Andrew, M. (2011). The Evolution of the Coverage of Islam and Muslim Cultures in Quebec Textbooks Contexts, The Journal of Educational Media, Memory, and Society. In M. Mc Andrew, F. Pingel et A. Triki-Yamani (éd.), Teaching about Islam and the Muslim World: Formal and Real Curriculum. 
Polly, S. (1992). The Portrayal of Jews and Judaism in Current Protestant Teaching Materials: A Context Analysis Ann Arbor, Michigan: University Microfilms International.

Potvin, M. et Carr, P. (2008). La valeur ajoutée de l'éducation antiraciste: conceptualisation et mise en oeuvre au Québec et en Ontario. Éducation et Francophonie, Mc Andrew, M. (dir.) rapports ethniques et éducation: perspectives nationales et internationales (XXXVI 1, printemps), 197-217.

Richler, M. (1992). Oh Canada! Oh Québec. Requiem pour un pays divisé. Candiac: Les Éditions Balzac.

Schoenfeld, S. (2010). Juifs au Canada. L'Encyclopédie canadienne.

Shahar, C., et Karpman, S. (2004). 2001 Census Analysis: The Jewish Community of Montreal. Montréal: CJA/UIA Federations Canada.

Shahar, C. et Karpman, S. (2006). Données statistiques sur les élèves fréquentant les écoles juives. Montréal: Association des écoles juives (rapport interne).

Sniderman, P.M., Northrup, D.A., Fletcher, J.F., Russel, P.H. et Tetlock, P.E. (1992). Working paper on antisemitisme in Québec. Institute for social research: York University, Ontario.

Tulchinsky, G. (1993). The Contours of Candian Jewish History. In R. J. Brym, Shaffir, William, et Weinfeld, Morton (éd.), The Jews In Canada (p. 5-21). Toronto: Oxford University Press.

Weinfeld, M. (1993). The jews of Quebec: an overview. In R. J. Brym, Shaffir, William, et Weinfeld, Morton (éd.), The Jews in Canada (p. 171-192). Toronto: Oxford University press.

Weinfeld, M. (2001). Like everyone else, but different: the paradoxical success of Canadian Jews. Toronto: McClelland et Stewart.

Weinfeld, M. (2008). Quebec Anti-Semitism and Anti-Semitism in Quebec. Jerusalem Center of Public Affaires (64). Document téléaccessible à l'adresse: $<$ http://jcpa.org/article/quebec-anti-semitism-and-anti-semitism-in-quebec/>. 


\section{Liste des manuels étudiés}

\section{Histoire et éducation à la citoyenneté, $2^{\mathrm{e}}$ cycle, $1^{\mathrm{re}}$ année:}

Bachand, Ch-A., Demers, S., Jean, G. et Poirier, P. (2007). Présences, sous la direction de Dalongeville, A., CEC.

Bédard, R. et Cardin, J-F. (avec Brodeur-Girard, S., Vanasse, C.) (2007). Le Québec: une histoire à suivre..., Grand Duc.

Fortin, S., Ladouceur, M., Larose, S. et Rose, F. (2007). Fresques, Chenelière Éducation.

Thibeault, A (avec Charland, J-P. et Ouellet, N. pour le tome A) (2007). Repères, Erpi.

\section{Histoire et éducation à la citoyenneté, $2^{\mathrm{e}}$ cycle, $2^{\mathrm{e}}$ année:}

Bachand, Ch-A., Demers, S., Jean, G. et Poirier, P. (2007). Présences, sous la direction de Dalongeville, A., CEC.

Brodeur-Girard, S., Carrière, S., Laverdière, M-H. et Vanasse, C. (avec Beauchamp, B. et Carrier, M.) (2008). Le Québec: une histoire à construire, Grand Duc.

Horguelin, C., Ladouceur, M., Lord, F. et Rose, F. (2007). Fresques, Chenelière Éducation.

Sarra-Bournet, M., Bourson, Y., Bégin, Y. et Gélinas, F. (2007). Repères, Erpi.

\section{Étique et culture religieuse, $1^{\text {er }}$ cycle du secondaire:}

Bélanger, D., Carrière, A., Després, P., Mainville, C. et Vachon, A-C. (2008). Etre en société, CEC.

Charbonneau, N-A. (sous la direction de Debunne, J-M.) (2008). Passeport pour la vie, Grand Duc.

Grondin, J., Lefebvre, S. et Weinstock, D. (2008). Tête-à-Tête, Grand Duc.

L'Hérault, B. et Sirois, C. (2010), Réflexions, Chenelière. 
Étique et culture religieuse, $2^{\mathrm{e}}$ cycle du secondaire:

Caron, G. et Garber, P. (avec Beaulieu, M-F.) (2008). Tête-à-Tête, Grand Duc.

Deraspe, S., Després, P., Fournier-Courcy, I. et Tardif, S. (2009). Tisser des liens, sous la direction de Jean Dansereau, CEC (2 vol.). 